\title{
Risk factors for early and late mortality after thoracic endovascular aortic repair
}

Ali Khoynezhad, MD, a Carlos E. Donayre, MD, ${ }^{b}$ Jennifer Smith, MD, ${ }^{b}$ George E. Kopchok, BS, ${ }^{b}$ Irwin Walot, MD, ${ }^{c}$ and Rodney A. White, MD

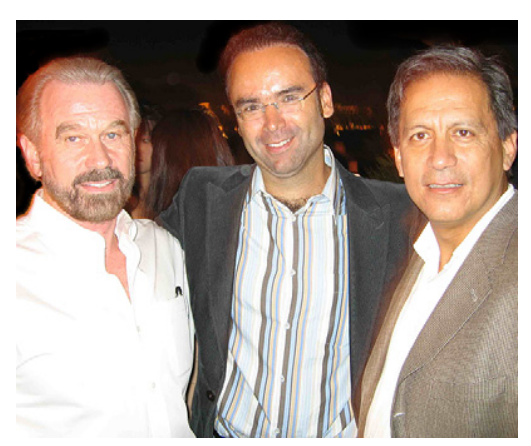

Drs White, Khoynezhad, and Donayre (left to right)
From the Section of Cardiovascular and Thoracic Surgery, University of Nebraska Medical Center, ${ }^{\mathrm{a}}$ Omaha, Neb; and Divisions of Vascular and Endovascular Surgery, ${ }^{b}$ and Interventional Radiology, ${ }^{c}$ Harbor-UCLA Medical Center, Torrance, Calif.

Carlos Donayre reports consulting fees, lecture fees, and grant support from Medtronic, and consulting fees from Gore. George Kopchok reports lecture fees from Medtronic. Rodney White reports consulting fees from Medtronic and Gore, and grant support from Medtronic.

Received for publication July 15, 2007; revisions received Aug 25, 2007; accepted for publication Sept 11, 2007.

Address for reprints: Ali Khoynezhad, MD, $\mathrm{PhD}$, Section for Thoracic and Cardiovascular Surgery, University of Nebraska Medical Center, 982315 Nebraska Medical Center, Omaha, NE 68198-2315 (E-mail: akhoynezhad@unmc.edu).

J Thorac Cardiovasc Surg 2008;135:1103-9 $0022-5223 / \$ 34.00$

Copyright (C) 2008 by The American Association for Thoracic Surgery

doi:10.1016/j.jtcvs.2008.02.001
Objective: The risk factors associated with death after thoracic endovascular aortic repair are poorly understood. The aim of this study is to analyze the risk factors associated with early and late mortality after thoracic endovascular aortic repair.

Methods: A total of 153 patients underwent 184 thoracic endovascular aortic repairs between 1998 and 2005. Prospectively collected data were entered into statistical software. Univariate and multivariate analyses were performed.

Results: The underlying pathologies included descending thoracic aortic aneurysm $(\mathrm{n}=91)$, acute type $\mathrm{B}$ aortic dissection $(\mathrm{n}=25)$, chronic type $\mathrm{B}$ aortic dissection $(n=42)$, aortic transection $(n=12)$, and penetrating aortic ulcer $(n=14)$. Thoracic endovascular aortic repair was technically successful in all but 3 patients. Another 3 patients required an open repair within the first month. Early and late mortality rates were $9.8 \%(\mathrm{n}=18)$ and $19 \%(\mathrm{n}=35)$ in a 16-month average period of follow-up, respectively. Type I procedural endoleak was the only significant predictor of early death in the multivariate model $(P=.0036$; odds ratio: $8.4 ; 95 \%$ confidence interval: 1.6-43.9). Multivariate Cox regression revealed chronic obstructive pulmonary disease $(P=.024$; odds ratio: 3.8 ; $95 \%$ confidence interval: $1.2-12.1)$, postoperative myocardial infarction $(P=.0053$; odds ratio: 9.7 ; $95 \%$ confidence interval: 2.0 48.4), and acute renal failure ( $P=.0006$; odds ratio: $22.8 ; 95 \%$ confidence interval: $3.8-137.6)$ to be independent risk factors for late mortality.

Conclusion: Procedural type I endoleak is an independent risk factor of early mortality after thoracic endovascular aortic repair. Chronic obstructive pulmonary disease, postoperative myocardial infarction, and acute renal failure are predictors of late death in the multivariate analysis.

$\mathrm{T}$ Thoracic endovascular aortic repair (TEVAR) is an emerging option to open repair in a selected population with aortic pathologies. Proposed advantages of TEVAR include shorter operative time, less blood loss, decreased need for general anesthesia, and shorter hospital stays. ${ }^{1-3}$ TEVAR avoids morbid thoracotomy and thoracoabdominal incisions, cardiopulmonary bypass, aortic crossclamping, and hypothermic circulatory arrest. A recent US multicenter trial with the Gore TAG thoracic endoprosthesis (WL Gore and Associates, Flagstaff, Ariz) involved patients with an underlying aneurysm who met anatomic eligibility criteria and were candidates for open surgical repair. ${ }^{4}$ The results of this study were encouraging and demonstrated feasibility and durability while reducing morbidity and mortality in early postoperative follow-up. ${ }^{4}$

The TAG trial, however, did not review and analyze the variables associated with death after TEVAR. To our knowledge, there are no published series on TEVAR that have focused on the risk factors of early versus late mortality. The aim of this study was to review our experience with TEVAR and to identify the risk factors of early and late mortality after TEVAR. 


\section{Abbreviations and Acronyms \\ CT = computed tomography \\ POD = postoperative day \\ TEVAR $=$ thoracic endovascular aortic repair}

\section{Patients and Methods}

A total of 153 consecutive patients were referred to HarborUCLA Medical Center for TEVAR between October of 1998 and September of 2005. A total of 184 procedures were performed on 117 male patients $(76.5 \%)$ and 67 female patients. After approval was obtained from the institutional review board, the patients were offered TEVAR through a single institution investigator Investigational Device Exemption approved by the Food and Drug Administration. All patients signed consent forms for the use of these investigational devices and agreed to participate in the surveillance protocols after deployment of the devices.

\section{Inclusion and Exclusion Criteria}

The inclusion criteria for TEVAR included all symptomatic aneurysms of the descending thoracic aorta, asymptomatic fusiform aneurysms of the descending thoracic aorta with at least twice the size or proximal normal aorta, saccular aneurysm greater than $5 \mathrm{~cm}$, complicated acute Stanford type B aortic dissections, aneurysmal degeneration (with a diameter at least twice the size or proximal normal aorta) of (or complicated) chronic Stanford type B aortic dissections, symptomatic penetrating aortic ulcers, and traumatic aortic transections. The term "complicated dissection" was defined as persistent/unrelenting back pain despite maximal medical therapy, uncontrollable hypertension, aortic enlargement more than $5 \mathrm{~mm}$ per year, malperfusion syndromes, or (imminent) rupture. Other inclusion criteria were signing the informed consent and agreeing to follow-up in the institutional surveillance program. Furthermore, the patient's arterial anatomy must have met device-specific requirements to be a candidate for TEVAR. Adequate proximal and distal landing zone and well-sized access vessels were evaluated before offering TEVAR to the patients.

The exclusion criteria included Stanford type A (or retro-A) aortic dissections, aneurysmal pathologies of ascending thoracic aorta, arterial anatomy unsuitable for TEVAR, patients with connective tissue disorder, age less than 18 years, pregnancy, systemic infection, and hypercoagulable disorder.

\section{Definition of Endoleaks}

Endoleaks were reported as defined as reporting standard by Chaikof and coworkers. ${ }^{5}$ Type I endoleaks are leaks from the proximal (Ia) or distal (Ib) landing zone. Type II endoleaks are not connected or associated to the landing zones or the junction between various stent grafts. Type III endoleaks are leaks between the junctions of 2 or more stent grafts. Type IV endoleaks are caused by graft wall porosity and are a problem with first-generation stent materials. Type $\mathrm{V}$ endoleak (endotension) is an increase in aneurysm diameter or pressure in the excluded sac without radiologic evidence of endoleak.

\section{Statistical Analysis}

Patient data, including demographics, risk factors, clinical symptoms, procedural details, computed tomography (CT) scans, angio- grams, postoperative complications, secondary interventions, and mortality, were collected in a retrospective manner by chart review and by review of prospective and concurrent Food and Drug Administration reports of the respective Investigational Device Exemption protocols. All data were entered into an electronic database. Statistical analysis was performed using the SPSS 13.0 for Windows statistical software package (SPSS Inc, Chicago, Ill). Fisher exact test and Cox regression were performed for univariate analysis of risk factors for early (within the first 30 days) and late mortality after TEVAR. Variables with $P$ values less than .05 in the univariate analysis were included in the stepwise model selection procedure of multivariate analysis. Multivariate logistic regression and Cox regression were used to evaluate the effects of a set of perioperative outcome variables on early and late death, and to establish independent risk factors. The actuarial survival was computed according to the Kaplan-Meier log-rank method. The statistical analysis underwent a mathematical review by a biostatistician from the University of Nebraska Medical Center.

The follow-up physical examination, contract CT, and laboratory work were performed according to the institutional surveillance protocol at 1, 6, and 12 months and yearly thereafter for 5 years. The protocol for postoperative imaging includes a 3-phase multidetector CT angiogram. A noncontrast scan through the chest and abdomen is followed by contrast computed tomographic angiography using $100 \mathrm{~mL}$ of nonionic contract. A 2-minute delayed CT scan is performed to enhance detection rate for endoleaks.

\section{Results}

A total of 153 consecutive patients (94 male, 59 female) underwent 184 endovascular procedures. This included primary and subsequent endovascular interventions. A total of 145 patients underwent endovascular repair using the Talent device (Medtronic, Minneapolis, Minn). Another 37 patients were treated with the Thoracic AneuRx graft (Medtronic), and an Excluder or TAG device (Gore, Flagstaff, Ariz) was deployed in the remaining 2 patients. The underlying pathology was descending or transverse aortic aneurysm in 91 patients (49\%), acute type B dissection in 25 patients (14\%), and chronic type B dissection in 42 patients $(23 \%)$. Twelve patients $(7 \%)$ had traumatic aortic transection, and 14 patients $(8 \%)$ were treated for penetrating aortic ulcer.

The majority of the patients were transferred from outside facilities. They were evaluated by cardiothoracic or vascular surgeons and deemed to be poor or nonoperative candidates. Some 35 of 153 patients (19\%) underwent emergency TEVAR for ruptured/leaking aorta, and 75 patients $(41 \%)$ had a previous major cardiac or aortic operation. Table 1 shows a selected list of the preoperative characteristics and comorbidities of this patient cohort. Figure 1 is the schematic representation of the distribution of proximal landing zones. Aortic pathologies involving the proximal or distal descending thoracic aorta were present in 127 and 51 patients, respectively. Four patients had aneurysms of the aortic arch, and 2 patients had type II thoracoabdominal aortic aneurysms. 
TABLE 1. Demographics of patients treated by thoracic endovascular aortic repair

\begin{tabular}{lc}
\hline \multicolumn{1}{c}{ Characteristics } & All patients (n = 184) \\
\hline Age (median): range 18-92 y & $71 \pm 13.7$ \\
s/p cardiac surgery & $18(10 \%)$ \\
s/p aortic surgery & $70(38 \%)$ \\
Ascending aortic & $5(3 \%)$ \\
Aortic arch & $8(4 \%)$ \\
Descending aorta & $23(13 \%)$ \\
Abdominal aorta & $34(18 \%)$ \\
Preoperative creatinine $(\mathrm{mg} / \mathrm{dL})$ & $1.5 \pm 1.7$ \\
Coronary artery disease & $49(26 \%)$ \\
Preoperative myocardial infarction & $4(2 \%)$ \\
Congestive heart failure & $16(7 \%)$ \\
Chronic obstructive pulmonary disease & $32(17 \%)$ \\
Peripheral vascular disease & $25(14 \%)$ \\
Preoperative stroke & $17(9 \%)$ \\
Diabetes mellitus & $17(9 \%)$ \\
Hypertension & $112(61 \%)$ \\
Obesity (body mass index $\left.\geq 30 \mathrm{~kg} / \mathrm{m}^{2}\right)$ & $8(4 \%)$ \\
\hline
\end{tabular}

\section{Technical Failures}

TEVAR was technically successful in $98 \%$ of the patients. In 3 patients, TEVAR was not achieved. The first patient, an 85year-old woman with aortic transection who was transferred via helicopter from another city, had aortic rupture in the endovascular suite before treatment. The second patient, an 85-year-old woman with a thoracic aneurysm, died of myocardial infarction on the operating table. The third patient, a 76-year-old woman with a 7-cm aneurysm of the descending thoracic aorta, had marginal access vessels. She was not a surgical candidate. Given her intraoperative access issues, the endovascular procedure was aborted without any harm to the patient.

Three other patients required open repair after undergoing TEVAR. All 3 patients underwent operation within the first month of TEVAR. All 3 patients survived the TEVAR/ open repair without any significant sequelae in the followup period. A 43-year-old man with multiple intra-abdominal and pelvic injuries had a transected aorta. The patient developed a localized dissection of the proximal landing zone with significant type I endoleak. He underwent immediate open graft replacement of the proximal descending thoracic aorta. He developed postoperative acute renal failure and respiratory failure but was discharged in satisfactory condition on postoperative day (POD) 39. A 66-year-old woman with lupus aortitis and a ruptured aneurysm of the mid-descending thoracic aorta underwent exclusion with a stent graft. Three weeks later, she required a reintervention for a newly diagnosed localized dissection at the proximal landing zone. A month after the first TEVAR procedure, she underwent the Bentall operation with total arch replacement for a retrograde dissection that was diagnosed in a follow-up CT scan. She

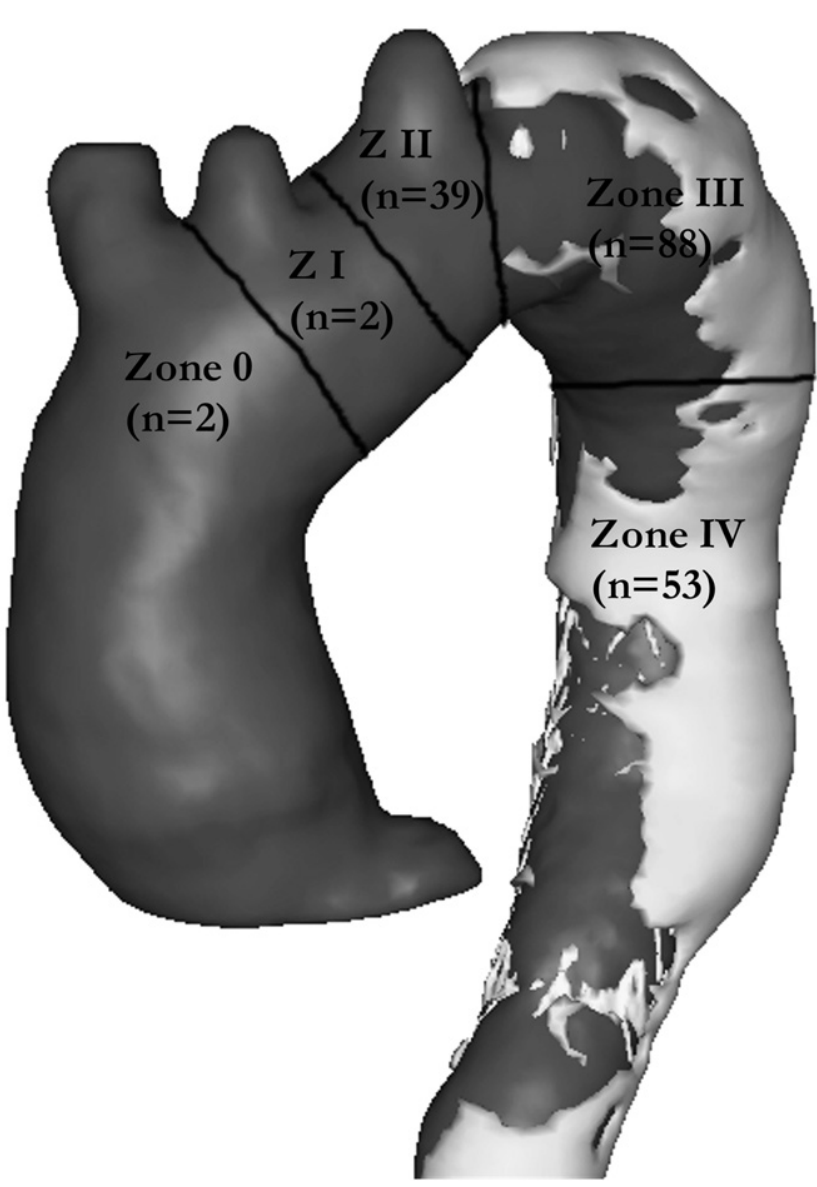

Figure 1. Proximal landing zones for all patients undergoing thoracic endovascular repair. Zone 0 : covering the innominate artery. Zone 1: covering the left carotid artery. Zone 2: covering the left subclavian artery. Zone 3 : involving proximal $1 / 3$ of the descending thoracic aorta. Zone 4 : involving distal $2 / 3$ of the descending thoracic aorta.

tolerated the procedure well and was discharged 10 days later. A 58-year-old woman with an aneurysmal degeneration of the chronic Stanford type B aortic dissection had a retrograde dissection into the ascending aorta requiring immediate ascending aortic replacement.

\section{Neurologic Deficits}

Postoperative paraplegia or paraparesis developed in 8 patients $(4.3 \%)$. Two patients recovered completely. Postoperative stroke developed in 8 other patients $(4.3 \%)$ with aneurysmal pathology. Two patients recovered completely. There was no correlation between the coverage of left subclavian artery and the incidence of posterior stroke. The analysis of risk factors associated with postoperative spinal cord injury and stroke has been discussed. ${ }^{6}$ The incidence of postoperative spinal cord injury, stroke, and 30-day mortality is summarized in Table 2. 
TABLE 2. Outcome of patients undergoing thoracic endovascular aortic repair based on underlying pathology

\begin{tabular}{lccc}
\hline \multicolumn{1}{c}{ Aortic pathology } & SCI (\%) & Stroke (\%) & $\begin{array}{c}\text { Early } \\
\text { mortality }\end{array}$ \\
\hline $\begin{array}{l}\text { Aortic aneurysm }(\mathrm{n}=91) \\
\text { Acute type B aortic }\end{array}$ & $8(8.8 \%)$ & $5(5.5 \%)$ & $9(9.9 \%)$ \\
$\quad$ dissection $(\mathrm{n}=25)$ & 0 & $1(4 \%)$ & $2(8 \%)$ \\
$\begin{array}{l}\text { Chronic type B dissection } \\
\quad(\mathrm{n}=42)\end{array}$ & 0 & $2(4.8 \%)$ & $5(11.9 \%)$ \\
$\begin{array}{l}\text { Aortic transection }(\mathrm{n}=12) \\
\text { Penetrating ulcer }(\mathrm{n}=14)\end{array}$ & 0 & 0 & $1(8.3 \%)$ \\
Total $(\mathrm{n}=184)$ & $8(4.3 \%)$ & $8(4.3 \%)$ & $18(9.8 \%)$ \\
\hline
\end{tabular}

$S C l$, Spinal cord injury.

\section{Early and Late Mortality}

Early mortality was $9.8 \%(18 / 184)$ in the entire cohort, and this was similar for patients with various aortic pathologies (Table 2). The causes of early death were myocardial infarction in 7 patients, retrograde dissection/cardiac tamponade in 3 patients (on PODs 2,3, and 8), sepsis/pneumonia in 3 patients, ruptured aorta in 2 patients ( 1 patient with traumatic transection before TEVAR and 1 patient with ruptured Kommerell diverticulum on POD 14 after carotid to bilateral subclavian bypass and zone 2 TEVAR), stroke in 1 patient (on POD 7), aortoesophageal fistula in 1 patient (on POD 5 after reintervention for TEVAR 5 months after initial TEVAR for aortoesophageal fistula), and aortobronchial fistula in 1 patient (recurrent hemorrhage on POD 9 after TEVAR). The causes of late mortality $(\mathrm{n}=19)$ were unknown in 6 patients $(2,6,31,46,47$, and 61 months after TEVAR), myocardial infarction in 5 patients $(2,2,4,8$, and 22 months after TEVAR), pneumonia in 4 patients $(3,3,22$, and 24 months after TEVAR), and cancer in 4 patients $(3,6,6$, and 13 months after TEVAR).

A univariate analysis perioperative variable was undertaken to determine the risk factors associated with early mortality (Table E1). The following variables were associated with early mortality: peripheral vascular disease, postoperative bleeding and transfusion, intraoperative hypotension, need for general anesthesia, procedural type I endoleak, postoperative myocardial infarction, vascular injury, and intensive care unit stay 2 days or more. The remainder of the other variables that were reviewed did not reveal any statistical significance. Multivariate logistic regression was used to calculate independent risk factors of early death after TEVAR. Procedural type I endoleak was the only significant predictor of early death in the multivariate model $(P=.0036)$. The patients with procedural endoleak are 8.4 times as likely to have early death as those without procedure endoleak (odds ratio $=8.4,95 \%$ confidence interval: $1.6-43.9$ ).

The overall 1- and 5-year survivals according to KaplanMeier were $80 \%$ and $67 \%$, respectively (Figure 2). Aorticrelated survival was $90 \%$ at 5 years (Figure 2). This was

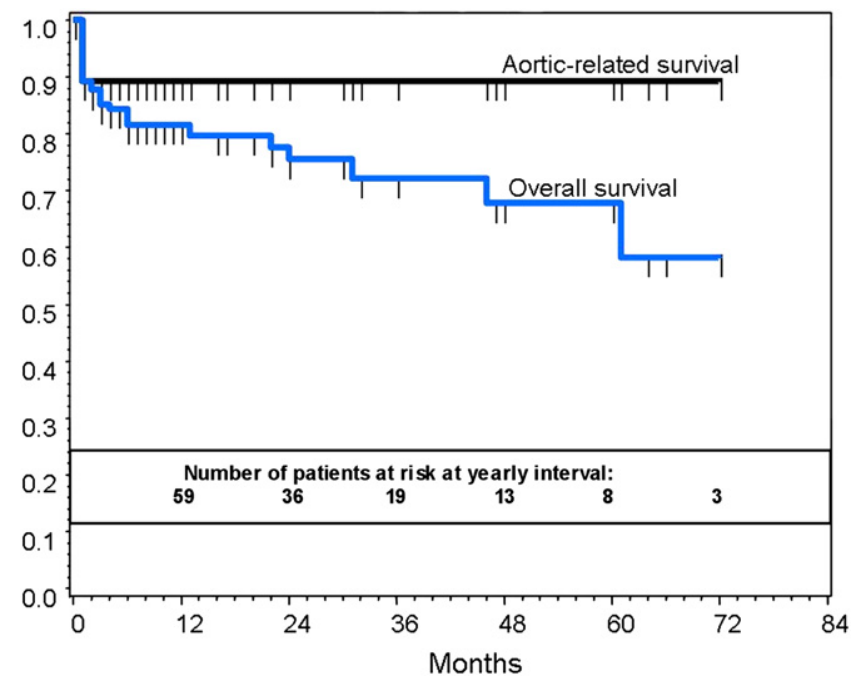

Figure 2. Kaplan-Meier survival curve comparing aortic-related (solid line) and overall mortality (gray line). At least 1 censored patient (tick marks) because of the length of the follow-up.

defined as all mortalities associated with aortic rupture and early mortality associated with the TEVAR procedure.

Table E2 contains the univariate analysis of variables associated with late mortality. The following risk factors of late mortality were identified: peripheral vascular disease, coronary artery disease, congestive heart failure, chronic obstructive pulmonary disease, postoperative myocardial infarction and acute renal failure, vascular injury, procedural blood loss of $400 \mathrm{~mL}$ or more, and intensive care unit stay of 2 days or more. Cox regression analysis revealed chronic obstructive pulmonary disease, postoperative myocardial infarction, and acute renal failure to be independent risk factors of mortality (Table 3 ).

\section{Endoleaks}

Procedural endoleaks were detected in $4.9 \%$ (9/184) of TEVARs. All were type I endoleaks. Three patients (3/9) had resolution of the endoleak in follow-up CT scans. One patient with traumatic transection required conversion to open repair (discussed previously). Three patients died of retrograde dissection/cardiac tamponade, stroke, and ruptured Kommerell's aneurysm on PODs 7, 8, and 14, respectively (discussed previously). Two patients (2/9) underwent reinterventions at 38 months and 66 months. One had successful resolution of the endoleak by endovascular approach. However, an 87-year-old woman with large chronic type B aortic dissection continues to have a small type Ia endoleak. She is not a candidate for open surgical repair to treat her endoleak.

The median follow-up was $16 \pm 17$ months (range 1-72 months) and was complete for $92 \%$ of the patients undergoing TEVAR. During this period of time, 36 late endoleaks 
TABLE 3. Multivariate predictors of overall survival (Cox regression)

\begin{tabular}{lccc}
\hline & Hazard ratio & $\mathbf{9 5 \%} \mathbf{C l}$ & $\boldsymbol{P}$ value \\
\hline $\begin{array}{l}\text { Chronic obstructive pulmonary } \\
\quad \text { disease }\end{array}$ & & & \\
No & 1 & - & .024 \\
$\quad$ Yes & 3.8 & $1.2-12.1$ & \\
$\begin{array}{l}\text { Postoperative myocardial infarction } \\
\text { No }\end{array}$ & 1 & - & .0053 \\
$\quad$ Yes & 9.7 & $2.0-48.4$ & \\
$\begin{array}{l}\text { Acute renal failure } \\
\text { No } \\
\text { Yes }\end{array}$ & 1 & - & .0006 \\
\hline Cl, Confidence interval. & 22.8 & $3.8-137.6$ & \\
\hline
\end{tabular}

(endoleaks after 30 days) were detected; 80.6\% (29/36) were type I endoleaks, $5.6 \%$ (2/36) were type II endoleaks, $11.1 \%$ (4/36) were type III endoleaks, and $2.8 \%(1 / 36)$ were type V endoleak. Twenty-three patients $(15 \%)$ required 34 of 184 $(18.5 \%)$ reinterventions in the follow-up (including the aforementioned 2 procedural endoleaks). Fifteen patients required only 1 reintervention, 5 patients required 2 reinterventions, and 3 patients required 3 reinterventions. No additional open operation was performed to treat endoleak beyond the first month.

\section{Discussion}

The prevalence of descending thoracic aortic aneurysm is estimated to be 10 in 10,000 elderly adults, with approximately $40 \%$ of these aneurysms confined to the thoracic aorta. ${ }^{7}$ Open repair of the descending and thoracoabdominal aortic and other aortic pathologies remains the gold standard. ${ }^{8-12}$ Endovascular repair is an emerging alternative to the open repair in a selected patient population. The patients in the current study had informed consent and were offered TEVAR for off-label indications through a single institution investigator Investigational Device Exemption approved by the Food and Drug Administration. The majority of these patients were not open surgical candidates, and TEVAR was the only possible treatment option.

The early survival of the patients undergoing TEVAR in this study is equal to the most historical groups undergoing open repair. ${ }^{8-12}$ The early mortality in the TAG study was $2.1 \%$ in the TEVAR group compared with $11.7 \%$ for the open repair control group for isolated "low-risk" descending thoracic aorta. ${ }^{13}$ All acute aortic syndromes with worse prognosis were excluded, and all included patients were deemed good surgical candidates assessed by 17 centers of excellence. The outcome of this series represents arguably the best assessment available of comparative real-world results for open repair of the descending thoracic aorta. Another comparative series of TEVAR versus the open repair comes from a study from the Massachusetts General Hospital. ${ }^{14}$ A total of 81 patients undergoing TEVAR were compared with 91 concurrent patients with open repair for the descending thoracic aorta or thoracoabdominal aortic aneurysm. All emergency/ruptured cases were excluded from the analysis. Early mortality was $8.9 \%$ versus $13.1 \%$ for the TEVAR and open control groups, respectively.

The long-term survival of patients undergoing TEVAR compared with open repair has been evaluated by few investigators. At the 54-month average follow-up, the actuarial survival was similar between the 2 groups in the trial from Massachusetts General Hospital. ${ }^{14}$ Similar findings were found by the TAG investigators, showing a 2-year survival of $78 \%$ and $76 \%$ for the TEVAR and open control groups, respectively. ${ }^{13}$ TEVAR seems to have no significant effect on the long-term survival of patients when compared with patients undergoing open repair. The 5-year survival was $67 \%$. This suggests that the long-term survival in the TEVAR group is not better than in the open repair group, despite a lower early mortality in the TEVAR group. Czerny and coworkers ${ }^{15}$ observed a $65 \%$ to $69 \%$ 5-year survival in patients undergoing TEVAR with an underlying aneurysm (excluding acute aortic syndromes), which is consistent with the results of current study.

TEVAR may reduce early mortality in this multimorbid patient population, but in the long-term follow-up, the patients will die of comorbidities such as cancer, coronary artery disease, chronic renal failure, and chronic obstructive pulmonary disease. The latter 3 variables were all independent risk factors of long-term mortality in the current study. Renal failure and chronic lung disease have been implicated with poor long-term outcome in patients undergoing open repair of the thoracic aortic pathologies. ${ }^{16}$ The association of coronary artery disease and cancer with poor long-term outcome needs no further discussion, because they represent the top 2 "killers" in the western countries.

Type I endoleak was the only independent risk factor for early mortality in this study. Careful intraoperative evaluation for endoleaks (intravascular ultrasound and multiplane "completion aortogram") is crucial to detect procedural endoleaks, because many are subtle and may be missed by just a regular aortogram. Given the prognostic significance of a procedural endoleak, it needs to be addressed aggressively in the endovascular suite. In many cases, there are endovascular options to treat type I endoleak. ${ }^{17}$ However, open conversion and repair should be offered to patients who have exhausted endovascular options. Furthermore, open surgical repair should be strongly considered in patients who are at significant risk for procedural type I endoleak. This reinforces the importance of comprehensive preoperative evaluation of the patients with aortic pathologies by an experienced group of physicians. These patients should be discussed in multidisciplinary clinics before offering them open or endovascular repair.

The endoleak rate in current study was $4.9 \%(9 / 184)$ in the first month and 43 (23.4\%) during the entire follow-up 
period. This includes recurrent (or persistent) endoleaks for the patients undergoing reintervention during the followup. Twenty-three patients (15\%) required reinterventions for ongoing type I or III endoleak, or type II/V endoleak with sac expansion. This endovascular strategy has been the mainstay of treatment of endoleaks after TEVAR. ${ }^{2,3,4,18-20}$ The rate of endoleak in the TAG trial was lower at $11 \%(12 / 110)$ and $9 \%(7 / 80) .{ }^{13}$ This is probably due to a selection bias. The TAG trial included a relatively healthy patient cohort with aneurysmal pathology who were open surgical candidates. In comparison, the endoleak rate was $29 \%$ in the other large studies by Czerny and colleagues ${ }^{15}$ and Parmer and colleagues. ${ }^{17}$ As in these series, the late endoleaks in this study were managed successfully in a nonsurgical fashion.

Because the procedural endoleak has a significant impact on the outcome, it is important to discuss the variables associated with procedural endoleak. Most important variables contributing to type I endoleak are modifiable. The most important risk factor for a procedural type I endoleak is an inadequate proximal or distal landing zone. ${ }^{15}$ Significant calcification or thrombus at the proximal/distal landing zone may impede complete apposition of the stent graft to the landing zone, thereby contributing to a type I endoleak. Therefore, patients with inadequate or poor quality proximal or distal landing zones need to be evaluated for surgical repair, if they are thought to be operative candidates. Endovascular repair should be offered to these patients with prohibitive operative risk if conservative management is not a reasonable option, and only after a detailed informed consent is obtained.

Furthermore, improper stent graft selection can contribute to procedural endoleak. Poor preoperative/intraoperative imaging and sizing of the proximal and distal landing zone can lead to under- or oversizing of the stent graft, both of which can contribute to endoleak. All these points underscore the importance of preoperative planning and experience in endovascular procedures. Surgeons should have at least 6 months of dedicated training before performing these procedures.

The length of the treated aorta and the number of stent grafts are also associated with an increased rate of endoleak. ${ }^{15,17}$ Type III endoleak is more common when the overlap between the multiple stent grafts is marginal. Other factors associated with procedural endoleak include the male gender and larger aneurysm size. ${ }^{17}$

There are several limitations to the current study. It represents a retrospective analysis of prospectively collected data as part of an investigational protocol. There is a lack of a risk-adjusted control group undergoing open repair for similar aortic pathologies. Furthermore, the follow-up is less than $100 \%$ complete, and the cause of death in 6 patients is undetermined in the follow-up. One may raise the argument that the cause of death in those 6 patients was related to the treated aortic pathology. Because the majority of these patients were transferred from other hospitals and cities, our efforts did not suc- ceed in tracking the patients who were lost to follow-up. A larger trial using a multicenter registry is recommended to substantiate the prognostic findings in this study and to establish the long-term ( $\geq 10$ years) durability and safety of the stent grafts.

\section{Conclusions}

This series demonstrates that type I procedural endoleak is the only significant predictor of early death in patients undergoing TEVAR. Superior imaging and careful preoperative planning may reduce the procedural endoleak. Multivariate analysis revealed chronic obstructive pulmonary disease, postoperative myocardial infarction, and acute renal failure to be independent risk factors for late death after TEVAR. All patients should be followed up closely to identify endoleaks and to establish the durability of stent grafts.

\section{References}

1. Song TK, Donayre CE, Walot I, Kopchok GE, Litwinski RA, Lippmann M, et al. Endograft exclusion of acute and chronic descending thoracic aortic dissections. J Vasc Surg. 2006;43:247-58.

2. Greenberg RK, O’Neill S, Walker E, Haddad F, Lyden SP, Svensson LG, et al. Endovascular repair of thoracic aortic lesions with the Zenith TX1 and TX2 thoracic grafts: intermediate-term results. J Vasc Surg. 2005;41:589-96.

3. Criado FJ, Abul-Khoudoud OR, Domer GS, McKendrick C, Zuzga M, Clark NS, et al. Endovascular repair of the thoracic aorta: lessons learned. Ann Thorac Surg. 2005;80:857-63.

4. Makaroun MS, Dillavou ED, Kee ST, Sicard G, Chaikof E, Bavaria J, et al. Endovascular treatment of thoracic aortic aneurysms: results of the phase II multicenter trial of the GORE TAG thoracic endoprosthesis. $J$ Vasc Surg. 2005;41:1-9.

5. Chaikof EL, Blankensteijn JD, Harris PL, White GH, Zarins CK, Bernhard VM, et al. Reporting standards for endovascular aortic aneurysm repair. J Vasc Surg. 2002;35:1048-60.

6. Khoynezhad A, Donayre CE, Bui H, Kopchok GE, Walot I, White RA. Risk factors of neurologic deficit after thoracic aortic endografting. Ann Thorac Surg. 2007;83:S882-9.

7. Clouse WD, Hallett JW Jr, Schaff HV, Gayari MM, Ilstrup DM, Melton LJ 3rd. Improved prognosis of thoracic aortic aneurysms: a population-based study. JAMA. 1998;280:1926-9.

8. Khoynezhad A, Bello R, Smego DR, Nwakanma L, Plestis KA. Improved outcome after repair of descending and thoracoabdominal aortic aneurysms using modern adjuncts. Interact CardioVasc Thorac Surg. 2005;4:574-6.

9. Estrera AL, Miller CC 3rd, Chen EP, Meada R, Torres RH, Porat EE, et al. Descending thoracic aortic aneurysm repair: 12-year experience using distal aortic perfusion and cerebrospinal fluid drainage. Ann Thorac Surg. 2005;80:1290-6.

10. Coselli JS, LeMaire SA, Conklin LD, Adams GJ. Left heart bypass during descending thoracic aortic aneurysm repair does not reduce the incidence of paraplegia. Ann Thorac Surg. 2004;77:1298-303.

11. Svensson LG, Crawford ES, Hess KR, Coselli JS, Safi HJ. Variables predictive of outcome in 832 patients undergoing repairs of the descending thoracic aorta. Chest. 1993;104:1248-53.

12. Kouchokos NT, Daily BB, Rokkas CK, Murphy SF, Bauer S, Abboud N. Hypothermic bypass and circulatory arrest for operations on the descending thoracic and thoracoabdominal aorta. Ann Thorac Surg. 1995;60:67-76.

13. Bavaria JE, Appoo JJ, Makaroun MS, Verter J, Yu ZF, Mitchell RS, et al. Endovascular stent grafting versus open surgical repair of descending thoracic aortic aneurysms in low-risk patients: a multicenter comparative trial. J Thorac Cardiovasc Surg. 2007;133:369-77.

14. Black JH3rd, Cambria RP. Current results of open surgical repair of descending thoracic aortic aneurysms. J Vasc Surg. 2006;43 (Suppl)A:6A-11A. 
15. Czerny M, Grimm M, Zimpfer D, Rodler S, Gottardi R, Hutschala D, et al. Results after endovascular stent graft placement in atherosclerotic aneurysms involving the descending aorta. Ann Thorac Surg. 2007;83: 450-5.

16. Cambria RP, Clouse WD, Davison JK, Dunn PF, Corey M, Dorer D. Thoracoabdominal aneurysm repair: results with 337 operations performed over a 15-year interval. Ann Surg. 2002;236:471-9.

17. Parmer SS, Carpenter JP, Stavropoulos SW, Fairman RM, Pochettino A, Woo EY, et al. Endoleaks after endovascular repair of thoracic aortic aneurysms. J Vasc Surg. 2006;44:447-52.
18. Morishita K, Kurimoto Y, Kawaharada N, Fukada J, Hachiro Y, Fujisawa $Y$, et al. Descending thoracic aortic rupture: role of endovascular stent-grafting. Ann Thorac Surg. 2004;78:1630-4.

19. Hansen CJ, Bui H, Donayre CE, Aziz I, Kim B, Kopchok GE, et al. Complications of endovascular repair of high-risk and emergent descending thoracic aortic aneurysms and dissections. I Vasc Surg. 2004;40:228-34.

20. Bortone AS, DeCillis E, D’Agostino D. Tupputi Schinosa LDL. Endovascular treatment of thoracic aortic disease four years of experience. Circulation. 2004;110(Suppl 2):II262-7. 
TABLE E1. Univariate predictors of early mortality (Fisher exact test)

\begin{tabular}{|c|c|c|c|}
\hline & $\begin{array}{c}\text { Alive at } 1 \text { mo } \\
\text { follow-up }\end{array}$ & $\begin{array}{c}\text { Early } \\
\text { Mortality }\end{array}$ & $\begin{array}{c}P \\
\text { value }\end{array}$ \\
\hline \multicolumn{4}{|l|}{ Age (y) } \\
\hline$<60$ & 37 & 4 & 1.0 \\
\hline$\geq 60$ & 96 & 12 & \\
\hline \multicolumn{4}{|l|}{ Gender } \\
\hline Male & 84 & 10 & 1.0 \\
\hline Female & 50 & 6 & \\
\hline \multicolumn{4}{|l|}{ ICU stay (d) } \\
\hline $0-1$ & 37 & 1 & .039 \\
\hline$\geq 2$ & 41 & 9 & \\
\hline \multicolumn{4}{|l|}{ Hospital stay (d) } \\
\hline $0-5$ & 41 & 3 & .51 \\
\hline$\geq 6$ & 50 & 7 & \\
\hline \multicolumn{4}{|l|}{ Pathology } \\
\hline Aneurysm & 65 & 8 & .97 \\
\hline Acute dissection & 21 & 2 & \\
\hline Chronic dissection & 24 & 4 & \\
\hline Transection & 11 & 1 & \\
\hline Penetrating ulcer & 13 & 1 & \\
\hline \multicolumn{4}{|l|}{ Rupture/leaking } \\
\hline No & 105 & 13 & 1.0 \\
\hline Yes & 29 & 3 & \\
\hline \multicolumn{4}{|l|}{ Coronary artery disease } \\
\hline No & 84 & 9 & .38 \\
\hline Yes & 34 & 6 & \\
\hline \multicolumn{4}{|l|}{ Hypertension } \\
\hline No & 34 & 4 & 1.0 \\
\hline Yes & 86 & 11 & \\
\hline \multicolumn{4}{|l|}{ Diabetes mellitus } \\
\hline No & 100 & 12 & 1.0 \\
\hline Yes & 16 & 1 & \\
\hline \multicolumn{4}{|l|}{$\begin{array}{l}\text { Acute myocardial } \\
\text { infarction }\end{array}$} \\
\hline No & 106 & 12 & .37 \\
\hline Yes & 3 & 1 & \\
\hline \multicolumn{4}{|l|}{ Congestive heart failure } \\
\hline No & 101 & 10 & .14 \\
\hline Yes & 10 & 3 & \\
\hline \multicolumn{4}{|l|}{ On hemodialysis } \\
\hline No & 105 & 11 & 1.0 \\
\hline Yes & 1 & 0 & \\
\hline \multicolumn{4}{|c|}{ Peripheral vascular disease } \\
\hline No & 93 & 7 & .019 \\
\hline Yes & 13 & 5 & \\
\hline \multicolumn{4}{|l|}{ Preoperative stroke } \\
\hline No & 96 & 10 & 1.0 \\
\hline Yes & 11 & 1 & \\
\hline \multicolumn{4}{|l|}{ Obesity } \\
\hline No & 73 & 10 & 1.0 \\
\hline Yes & 7 & 0 & \\
\hline \multicolumn{4}{|l|}{ Smoking } \\
\hline No & 41 & 3 & .25 \\
\hline Yes & 62 & 11 & \\
\hline
\end{tabular}

TABLE E1. Continued

Alive at 1 mo Early $P$

follow-up Mortality value

Chronic obstructive pulmonary disease

$$
\text { No }
$$

Yes

Operating time (min)

$$
\begin{aligned}
& <180 \\
& \geq 180
\end{aligned}
$$

Procedural urine output

$$
<500
$$

$\geq 500$

Blood loss (mL)

$$
<400
$$$$
\geq 400
$$

Contrast use $(\mathrm{mL})$

$$
<100
$$

\begin{tabular}{|c|c|c|}
\hline 85 & 6 & .068 \\
\hline 23 & 6 & \\
\hline 48 & 3 & .30 \\
\hline 41 & 6 & \\
\hline 52 & 4 & .34 \\
\hline 42 & 7 & \\
\hline 50 & 3 & .22 \\
\hline 58 & 9 & \\
\hline 38 & 2 & .44 \\
\hline 42 & 5 & \\
\hline 46 & 3 & .17 \\
\hline 32 & 6 & \\
\hline 45 & 3 & .52 \\
\hline 62 & 7 & \\
\hline 37 & 4 & .41 \\
\hline 44 & 2 & \\
\hline 72 & 5 & .044 \\
\hline 39 & 9 & \\
\hline 61 & 4 & .025 \\
\hline 5 & 3 & \\
\hline 58 & 7 & .72 \\
\hline 26 & 2 & \\
\hline 74 & 8 & 1.0 \\
\hline 7 & 0 & \\
\hline 104 & 12 & .74 \\
\hline 26 & 4 & \\
\hline 69 & 7 & .65 \\
\hline 15 & 1 & \\
\hline 21 & 4 & \\
\hline 65 & 8 & 1.0 \\
\hline 53 & 6 & \\
\hline
\end{tabular}$$
\geq 100
$$

Fluoroscopy time (min)

$$
<8
$$$$
\geq 8
$$

Neck diameter (mm)

$$
\begin{aligned}
& <31 \\
& \geq 31
\end{aligned}
$$

Neck length (mm)

$$
<30
$$$$
\geq 30
$$

General anesthesia

$$
\text { No }
$$

Yes

Intraoperative hypotension

No

Use of adenosine for cardiac arrest No

Yes

Cerebrospinal fluid drain

$$
\begin{aligned}
& \text { No } \\
& \text { Yes }
\end{aligned}
$$

Iliac artery conduit

No

Yes

Brachial/radial access

None

Brachial artery

Radial artery

No. of stent grafts

$$
\begin{aligned}
& <3 \\
& \geq 3
\end{aligned}
$$

Subclavian artery

Covered

No
Yes
anding zone
$0-2$
$3-4$

$\begin{array}{rll}74 & 6 & .11 \\ 27 & 6 & \\ 32 & 7 & .13 \\ 102 & 9 & \end{array}$


TABLE E1. Continued

Alive at 1 mo Early $P$

follow-up Mortality value

Internal iliac artery coverage

No

Yes

Procedural endoleak

None

Type 1

Endoleak in follow-up

0

1

2

3

Postoperative myocardial infarction

No

Yes

Packed red blood units

0

$\geq 1$

Postoperative bleeding

No

Yes

Infection

No

Yes

Vascular injury

No

Yes

Distal embolization

No

Yes

Vascular thrombosis

No

Yes

Acute renal failure

No

Yes

Stent graft kink

No

$\begin{array}{ll}79 & 8\end{array}$

1.0

50

$84 \quad 6$

.024

53

$\begin{array}{lll}69 & 10 & 1.0\end{array}$

$19 \quad 2$

$1 \quad 0$

30

$\begin{array}{lll}78 & 8 & .001\end{array}$

35

$\begin{array}{lll}60 & 3 & .0098\end{array}$

$17 \quad 6$

$\begin{array}{lll}78 & 9 & .015\end{array}$

23

Yes

$\begin{array}{rrr}70 & 9 & .65 \\ 11 & 2 & \\ 77 & 7 & .0063 \\ 4 & 4 & \\ & & \\ 78 & 10 & .40 \\ 3 & 1 & \\ 79 & 10 & .12 \\ 0 & 1 & \\ 76 & 9 & .064 \\ 5 & 3 & \\ 80 & 11 & 1.0 \\ 1 & 0 & \end{array}$

ICU, Intensive care unit. 
TABLE E2. Univariate predictors of overall survival (Cox regression)

\begin{tabular}{|c|c|c|c|}
\hline & Hazard ratio & $95 \% \mathrm{CI}$ & $P$ value \\
\hline \multicolumn{4}{|l|}{ Age (y) } \\
\hline$<60$ & 1 & - & .23 \\
\hline$\geq 60$ & 1.9 & $0.7-5.5$ & \\
\hline \multicolumn{4}{|l|}{ Gender } \\
\hline Male & 1 & - & .34 \\
\hline Female & 1.4 & $0.7-2.9$ & \\
\hline \multicolumn{4}{|l|}{ ICU stay } \\
\hline $0-1$ & 1 & - & .026 \\
\hline$\geq 2$ & 3.2 & $1.1-8.8$ & \\
\hline \multicolumn{4}{|l|}{ Hospital stay } \\
\hline $0-5$ & 1 & - & .20 \\
\hline$\geq 6$ & 0.8 & $0.6-1.1$ & \\
\hline \multicolumn{4}{|l|}{ Pathology } \\
\hline Aneurysms & 1.3 & $0.7-2.7$ & .43 \\
\hline Others & 1 & - & \\
\hline \multicolumn{4}{|c|}{ Rupture/leaking } \\
\hline & 1 & - & .48 \\
\hline & 0.7 & $0.3-1.8$ & \\
\hline \multicolumn{4}{|c|}{ Coronary artery disease } \\
\hline No & 1 & - & .0067 \\
\hline Yes & 2.7 & $1.3-5.6$ & \\
\hline \multicolumn{4}{|l|}{ Hypertension } \\
\hline No & 1 & - & .98 \\
\hline Yes & 1.0 & $0.4-2.2$ & \\
\hline \multicolumn{4}{|c|}{ Diabetes mellitus } \\
\hline No & 1 & - & .49 \\
\hline Yes & 1.4 & $0.5-3.7$ & \\
\hline \multicolumn{4}{|c|}{ Acute myocardial infarction } \\
\hline No & 1 & - & .20 \\
\hline Yes & 2.6 & $0.6-10.9$ & \\
\hline \multicolumn{4}{|c|}{ Congestive heart failure } \\
\hline No & 1 & - & .0068 \\
\hline Yes & 3.3 & $1.4-7.9$ & \\
\hline \multicolumn{4}{|c|}{ Peripheral vascular disease } \\
\hline No & 1 & - & .009 \\
\hline Yes & 3.1 & $1.3-7.5$ & \\
\hline \multicolumn{4}{|c|}{ Preoperative stroke } \\
\hline No & 1 & - & .53 \\
\hline Yes & 0.6 & $0.1-2.7$ & \\
\hline \multicolumn{4}{|l|}{ Obesity } \\
\hline No & 1 & - & .42 \\
\hline Yes & 0.4 & $0.1-3.3$ & \\
\hline \multicolumn{4}{|l|}{ Smoking } \\
\hline No & 1 & - & .17 \\
\hline Yes & 1.8 & $0.8-4.3$ & \\
\hline \multicolumn{4}{|c|}{$\begin{array}{l}\text { Chronic obstructive pulmonary } \\
\text { disease }\end{array}$} \\
\hline No & 1 & - & .0045 \\
\hline Yes & 3.2 & $1.4-7.1$ & \\
\hline \multicolumn{4}{|c|}{ Operating time (min) } \\
\hline$<180$ & 1 & - & .13 \\
\hline$\geq 180$ & 2.1 & $0.8-5.2$ & \\
\hline
\end{tabular}

TABLE E2. Continued

\begin{tabular}{|c|c|c|c|}
\hline & Hazard ratio & $95 \% \mathrm{Cl}$ & $P$ value \\
\hline \multicolumn{4}{|c|}{ Procedural urine output } \\
\hline$<500$ & 1 & - & .092 \\
\hline$\geq 500$ & 2.1 & $0.9-5.0$ & \\
\hline \multicolumn{4}{|l|}{ Blood loss (mL) } \\
\hline$<400$ & 1 & - & .039 \\
\hline$\geq 400$ & 2.6 & $1.1-6.5$ & \\
\hline \multicolumn{4}{|l|}{ Contrast use (mL) } \\
\hline$<100$ & 1 & - & .44 \\
\hline$\geq 100$ & 1.5 & $0.5-4.5$ & \\
\hline \multicolumn{4}{|c|}{ Fluoroscopy time (min) } \\
\hline$<8$ & 1 & - & .13 \\
\hline$\geq 8$ & 2.1 & $0.8-5.5$ & \\
\hline \multicolumn{4}{|c|}{ Neck diameter (mm) } \\
\hline$<31$ & 1 & - & .12 \\
\hline$\geq 31$ & 2.1 & $0.8-5.4$ & \\
\hline \multicolumn{4}{|l|}{ Neck length (mm) } \\
\hline$<30$ & 1 & - & .38 \\
\hline$\geq 30$ & 0.6 & $0.2-1.7$ & \\
\hline \multicolumn{4}{|l|}{ General anesthesia } \\
\hline No & 1 & - & .27 \\
\hline Yes & 1.6 & $0.7-3.5$ & \\
\hline \multicolumn{4}{|c|}{ Intraoperative hypotension } \\
\hline No & 1 & - & .23 \\
\hline Yes & 2.2 & $0.6-8.0$ & \\
\hline \multicolumn{4}{|c|}{$\begin{array}{l}\text { Use of adenosine for } \\
\text { cardiac } \\
\text { arrest }\end{array}$} \\
\hline No & 1 & - & .48 \\
\hline Yes & 0.6 & $0.2-2.3$ & \\
\hline \multicolumn{4}{|c|}{ Iliac artery conduit } \\
\hline No & 0 & - & .77 \\
\hline Yes & 0.9 & $0.3-2.3$ & \\
\hline \multicolumn{4}{|c|}{ Brachial/radial access } \\
\hline Brachial artery & 1 & - & .78 \\
\hline Radial artery & 1.2 & $0.3-5.2$ & \\
\hline \multicolumn{4}{|l|}{ No. of stent grafts } \\
\hline$<3$ & 1 & - & .36 \\
\hline$\geq 3$ & 1.4 & $0.7-3.1$ & \\
\hline \multicolumn{4}{|l|}{ Subclavian artery } \\
\hline \multicolumn{4}{|l|}{ Covered } \\
\hline No & 1 & - & .16 \\
\hline Yes & 1.9 & $0.8-4.6$ & \\
\hline \multicolumn{4}{|l|}{ Landing zone } \\
\hline $0-2$ & 1 & - & .19 \\
\hline $3-4$ & 0.6 & $0.3-1.3$ & \\
\hline \multicolumn{4}{|c|}{ Internal iliac artery coverage } \\
\hline No & 1 & - & .87 \\
\hline Yes & 0.8 & $0.1-6.4$ & \\
\hline \multicolumn{4}{|c|}{ Procedural endoleak } \\
\hline None & 1 & - & .10 \\
\hline Type 1 & 2.8 & $0.8-9.8$ & \\
\hline \multicolumn{4}{|c|}{ Endoleak in follow-up } \\
\hline 0 & 1 & - & .12 \\
\hline $1-3$ & 0.4 & $0.1-1.3$ & \\
\hline
\end{tabular}


TABLE E2. Continued

\begin{tabular}{|c|c|c|c|}
\hline & Hazard ratio & $95 \% \mathrm{Cl}$ & $P$ value \\
\hline \multicolumn{4}{|c|}{$\begin{array}{l}\text { Postoperative myocardial } \\
\text { infarction }\end{array}$} \\
\hline No & 1 & - & $<.001$ \\
\hline Yes & 8.8 & $3.6-21.6$ & \\
\hline \multicolumn{4}{|c|}{ Packed red blood units } \\
\hline 0 & 1 & - & .22 \\
\hline$\geq 1$ & 1.7 & $0.7-4.1$ & \\
\hline \multicolumn{4}{|c|}{ Postoperative bleeding } \\
\hline No & 1 & - & .014 \\
\hline Yes & 4.9 & $1.4-17.6$ & \\
\hline \multicolumn{4}{|c|}{ Infection } \\
\hline No & 1 & - & .29 \\
\hline Yes & 1.8 & $0.6-5.5$ & \\
\hline \multicolumn{4}{|c|}{ Vascular injury } \\
\hline No & 1 & - & .0058 \\
\hline Yes & 4.2 & $1.5-11.8$ & \\
\hline \multicolumn{4}{|c|}{ Distal embolization } \\
\hline No & 1 & - & .16 \\
\hline Yes & 2.9 & $0.7-12.5$ & \\
\hline \multicolumn{4}{|c|}{ Vascular thrombosis } \\
\hline No & 1 & - & .038 \\
\hline Yes & 8.8 & $1.1-68.7$ & \\
\hline \multicolumn{4}{|c|}{ Acute renal failure } \\
\hline No & 1 & - & .023 \\
\hline Yes & 3.7 & $1.2-11.2$ & \\
\hline
\end{tabular}

$\mathrm{Cl}$, Confidence interval; ICU, intensive care unit. 